\title{
Thermal Operator Representation of Feynman Graphs
}

\author{
Ashok Das \\ Department of Physics and Astronomy, University of Rochester, Rochester, NY 14627, USA and \\ Saha Institute of Nuclear Physics, 1/AF Bidhannagar, Calcutta 700064, INDIA
}

Received on 11 August, 2006

\begin{abstract}
In this talk I describe an interesting relation between Feynman graphs at finite temperature and chemical potential and the corresponding ones at zero temperature. The operator relating the two which we call the "thermal operator", simplifies the evaluation of finite temperature graphs and helps in understanding better several physical questions such as cutting rules, forward scattering, gauge invariance etc at finite temperature.
\end{abstract}

Keywords: Quantum field theory; Finite temperature and thermal operator

\section{INTRODUCTION}

Quantum field theories at zero temperature have been studied in great detail over many decades in the past. As a result, we understand many features of the zero temperature quantum field theory at least perturbatively. The perturbative calculations are carried out through the use of Feynman diagrams and such calculations with necessary regularizations are fairly routine. Calculations in quantum field theroies at finite temperature and chemical potential can also be carried out through the use of finite temperature Feynman graphs, However, such calculations prove a lot more challenging than the zero temperature counterparts and, as a result, have not yet been embraced by the community at large and there is a continuous effort to simplify such calculations. In this talk I will describe how a simple relation exists between Feynman graphs at finite temperature and the corresponding ones at zero temperature through a "thermal operator". As a result of this relation, calculations at finite temperature can be simplified. But, more importantly, such a relation allows one to better understand various physical issues such as cutting rules, forward scattering amplitude, gauge invariance, divergence structure etc at finite temperature. This talk is based on a series of papers that we have written on the subject [1-4] where references to other relevant papers may be found.

The talk is organized as follows. In section II, I will briefly review the three formalisms that are commonly used to describe finite temperature quantum systems. In this section, I will develop the notion of the thermal operator within the context of the imaginary time formalism within the context of a massive real scalar field. I will also describe various properties of the thermal operator as well as the meaning of such a relation. In section III, I will develop the idea of the thermal operator in the closed time path formalism (real time formalism). The thermal operator representation is the most direct in this formalism and I will discuss the thermal operator representation for theories involving massive, real scalar field, a massive fermion (without a chemical potential) as well as the Yang-Mills theory. In section IV, I will describe the thermal operator representation for a fermion with a chemical potential and give some results on how calculations at finite temperature and finite chemical potential, in the case of QED, lead to the result that the chemical potential is shifted by radiative corrections. Finally, I will close with some observations on possible future work in section $\mathbf{V}$.

\section{THERMAL OPERATOR REPRESENTATION IN THE IMAGINARY TIME FORMALISM}

At zero temperature, a given quantum field theory has a set of causal propagators (Green's functions) and interaction vertices with which one can construct and calculate any Feynman graph. Each Feynman graph, of course, represents a radiative (quantum) correction to a given process and the calculation of the Feynman graphs are best carried out in the energy-momentum space. At finite temperature, the quantum system can also be described by a quantum field theory. However, the boundary conditions at finite temperature are different from the ones at zero temperature. Correspondingly the propagators (Green's functions) of the theory modify and become functions of temperature to reflect the boundary conditions although the interaction vertices have the same structure as at zero temperature (and do not have any temperature dependence).

There are basically three equivalent and commonly used formalisms to describe quantum systems in equilibrium at finite temperature. The oldest of the formalisms is known as the "Imaginary Time Formalism" [5-8] and is based on the simple observation that if we let

$$
t \rightarrow-\frac{i}{T}=-i \beta
$$

where $T$ represents the equilibrium temperature, then the time evolution operator becomes

$$
e^{-i H t} \rightarrow e^{-\beta H},
$$

where we have set the Boltzmann constant to unity for simplicity. This is, of course, reminiscent of the density matrix of a quantum statistical system described by the Hamiltonian $H$ and correspondingly one can define the partition function for the system in a straightforward manner.

Since in this formalism the time variable is rotated to the (negative) imaginary axis, such a description of a quantum system at finite temperature is commonly known as the imaginary time formalism. In this formalism, therefore, one deals 


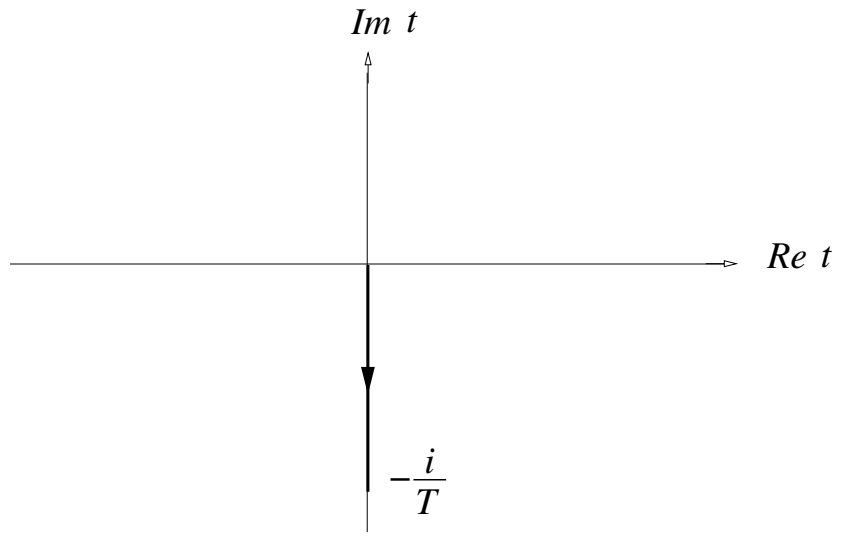

FIG. 1: Time contour in the imaginary time formalism.

with an Euclidean field theory. However, since time takes values over a finite interval

$$
0 \leq \tau \leq \beta
$$

where $\tau$ represents the time along the negative imaginary axis, the conjugate energy variable takes only discrete values

$$
p_{0}=\left\{\begin{array}{cl}
2 k i \pi T & \text { for bosons } \\
(2 k+1) i \pi T & \text { for fermions }
\end{array}\right.
$$

where $k$ is an integer. These energy values are commonly known as the Matsubara frequencies. As a result, for a real, massive scalar field, for example, the propagator will have the form of an Euclidean propagator

$$
\Delta^{(T)}(p)=\frac{1}{(2 \pi k T)^{2}+E^{2}}, \quad E=\sqrt{\vec{p}^{2}+m^{2}},
$$

with the energy taking discrete values given by the Matsubara frequencies. In a scalar field theory, one can now calculate Feynman graphs with this propagator and the interaction vertices for the Euclidean field theory to determine quantum corrections at finite temperature. However, since energy now takes discrete values, in loops we have to replace the integration over the loop energies to summation over internal Matsubara frequencies. Summation over these integers at higher loops presents a nontrivial challenge in this formalism.

In the imaginary time formalism, as we have seen, the time variable is traded in for temperature. However, one can also define a formalism to describe a quantum system at finite temperature where both time and temperature are present. This known as the real time formalism (there are two such formalisms that are commonly in use). The price one has to pay for this is to have an elaborate time contour in the complex time plane as shown in Fig. 2. Here $\sigma$ is a real parameter taking values in the interval $0 \leq \sigma \leq 1$. For $\sigma=0$, the real time formalism is due to Schwinger known as the closed time path formalism [8-10] while for $\sigma=\frac{1}{2}$, the real time formalism corresponds to thermofield dynamics $[8,11]$ of Umezawa

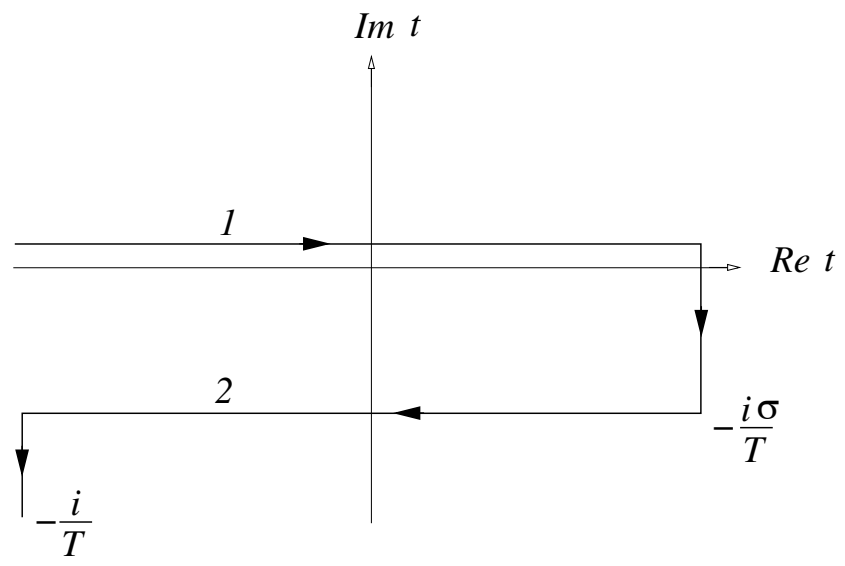

FIG. 2: Time contour in the real time formalisms.

et. al. Thermofield dynamics is quite useful in understanding questions involving the Hilbert space structure of a theory while the closed time path formalism is quite versatile and is useful even in studies of certain kinds of nonequilibrium phenomena.

In the real time formalisms, since time takes values over the entire real interval (the contributions from imaginary branches decouple since they lie at $\operatorname{Re} t \rightarrow \pm \infty$ ) much like in the zero temperature theory, energy has a continuous spectrum. However, now there are two real branches of the time contour leading to a necessity for doubling the field degrees of freedom. Thus, if we denote the two field degrees for a massive real scalar field in the closed time path formalism as $\phi_{ \pm}$, we see that the propagator in such a case will be a $2 \times 2$ matrix of the form

$$
\Delta^{(T)}(p)=\left(\begin{array}{cc}
\Delta_{++}^{(T)}(p) & \Delta_{+-}^{(T)}(p) \\
\Delta_{-+}^{(T)}(p) & \Delta_{--}^{(T)}(p)
\end{array}\right),
$$

with $\left(n\left(\left|p_{0}\right|\right)\right.$ is the $\mathrm{BE}$ distribution function)

$$
\begin{aligned}
\Delta_{++}^{(T)}(p) & =\frac{i}{p^{2}-m^{2}+i \varepsilon}+2 \pi n\left(\left|p_{0}\right|\right) \delta\left(p^{2}-m^{2}\right), \\
\Delta_{+-}^{(T)}(p) & =2 \pi\left(\theta\left(-p_{0}\right)+n\left(\left|p_{0}\right|\right)\right) \delta\left(p^{2}-m^{2}\right), \\
\Delta_{-+}^{(T)}(p) & =2 \pi\left(\theta\left(p_{0}\right)+n\left(\left|p_{0}\right|\right)\right) \delta\left(p^{2}-m^{2}\right), \\
\Delta_{--}^{(T)}(p) & =-\frac{i}{p^{2}-m^{2}-i \varepsilon}+2 \pi n\left(\left|p_{0}\right|\right) \delta\left(p^{2}-m^{2}\right) .
\end{aligned}
$$

The problem of summation over integer energy values of the imaginary time formalism is here replaced by the doubled degrees of freedom. This leads to many more Feynman diagrams for a given process at any higher loop involving products of highly singular delta functions. This brief review of the different formalisms describing quantum field theories at finite temperature is simply meant to illustrate the challenges facing any calculation of Feynman graphs at finite temperature. This also makes it clear that if there exists a simple relation between a Feynman graph at finite temperature and the corresponding one at zero temperature, that will be of immense 
use for a variety of reasons as indicated in the introduction. In this section, we will describe such a relation in the imaginary time formalism before moving on to the real time formalism (where the relation is much simpler) in the remaining sections.

Although at zero temperature, the computation of Feynman diagrams is conventionally carried out in the energymomentum space, for the purpose of obtaining a thermal operator representation, it is most useful to work in the mixed space where the energy variable is Fourier transformed so that the propagator is a function of time and the spatial component of momentum. Such a (mixed space) representation is quite useful in many studies at finite temperature. We note that if we Fourier transform the scalar propagator (5) in the energy variable, we obtain

$$
\begin{aligned}
& \Delta^{(T)}(\tau, E)=T \sum_{k} \frac{e^{-i 2 \pi k T \tau}}{(2 \pi k T)^{2}+E^{2}} \\
= & \frac{1}{2 E}\left[(\theta(\tau)+n(E)) e^{-E \tau}+(\theta(-\tau)+n(E)) e^{E \tau}\right] \\
= & (1+n(E)(1-S(E))) \Delta^{(T=0)}(\tau, E) \\
= & O^{(T)}(E) \Delta^{(T=0)}(\tau, E), \quad-\frac{1}{T} \leq \tau \leq \frac{1}{T} .
\end{aligned}
$$

Here $\tau$ is the Euclidean time and $n(E)$ represents the BoseEinstein distribution function

$$
n(E)=\frac{1}{e^{\beta E}-1} .
$$

This is a remarkable result which shows that in the mixed space, the thermal propagator in the imaginary time formalism can be separated nicely into a zero temperature part and a finite temperature part much like in the real time formalism (see (7)). Furthermore, it shows that the thermal propagator can be factorized such that it is related to the zero temperature propagator through the basic thermal operator

$$
O^{(T)}(E)=1+n(E)(1-S(E))
$$

which is independent of the time variable. This basic factorization of the thermal propagator is really at the heart of the thermal operator representation for any finite temperature Feynman graph (the proof of which is most direct in the closed time path formalism). For example, let us note that given the factorization of the basic propagator of the scalar field theory, any finite temperature Feynman diagram where all vertices are external will factorize. For example, in a $\phi^{3}$ theory the one loop graph the $N$-point graph with all vertices external (see Fig. 3) is easily seen to factorize as follows.

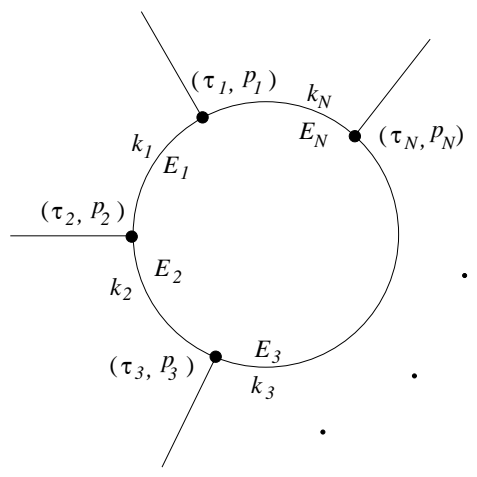

FIG. 3: One loop $N$-point graph in $\phi^{3}$ theory that is easily seen to factorize.

$$
\begin{aligned}
\Gamma_{N}^{(T)}= & \int \prod_{i=1}^{N} \mathrm{~d}^{3} k_{i} \delta^{(3)}\left(k_{i}-k_{i+1}+p_{i+1}\right) \gamma_{N}^{(T)} \\
= & \int \prod_{i=1}^{N} \mathrm{~d}^{3} k_{i} \delta^{(3)}\left(k_{i}-k_{i+1}+p_{i+1}\right) \\
& \times \Delta^{(T)}\left(\tau_{1}-\tau_{2}, E_{1}\right) \cdots \Delta^{(T)}\left(\tau_{N}-\tau_{1}, E_{N}\right) \\
= & \int \prod_{i=1}^{N} \mathrm{~d}^{3} k_{i} \delta^{(3)}\left(k_{i}-k_{i+1}+p_{i+1}\right) \\
& \times \prod_{i=1}^{N}\left(1+n_{i}\left(1-S_{i}\right)\right) \\
& \times \Delta^{(T=0)}\left(\tau_{1}-\tau_{2}, E_{1}\right) \cdots \Delta^{(T=0)}\left(\tau_{N}-\tau_{1}, E_{N}\right) \\
= & \int \prod_{i=1}^{N} \mathrm{~d}^{3} k_{i} \delta^{(3)}\left(k_{i}-k_{i+1}+p_{i+1}\right) O^{(T)} \gamma_{N}^{(T=0)},(12
\end{aligned}
$$

where $n_{i}=n\left(E_{i}\right), S_{i}=S\left(E_{i}\right)$ and we have identified

$$
O^{(T)}=\prod_{i=1}^{N} O^{(T)}\left(E_{i}\right)=\prod_{i=1}^{N}\left(1+n_{i}\left(1-S_{i}\right)\right) .
$$

This shows for simple graphs that if we evaluate the zero temperature graph, the value of the finite temperature graph can be obtained from it by operating with the thermal operator (13). The integration over the spatial momenta, of course, need to be done. However, the summation of Matsubara frequencies has already been taken care of.

Such a simple factorization of graphs is easily seen to hold even at higher loops if the graph involves only external vertices. For example, the same line of analysis as done above can be carried out for the two loop graphs shown in Fig. 4 in the $\phi^{4}$ theory. However, the extension of this relation to graphs with internal time vertices (sse Fig. 5) is not at all obvious. The difficulty arises because the internal time coordinates of a graph have to be integrated. At finite temperature, the range of integration in the imaginary time formalism, as we have already noted, is given by

$$
\int_{0}^{\frac{1}{T}} \mathrm{~d} \tau
$$



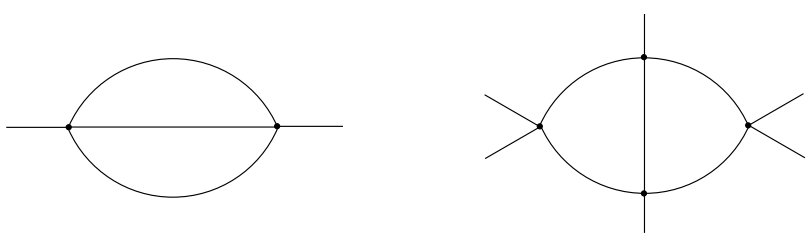

FIG. 4: Some two loop graphs in the $\phi^{4}$ theory that are easily seen to factorize.
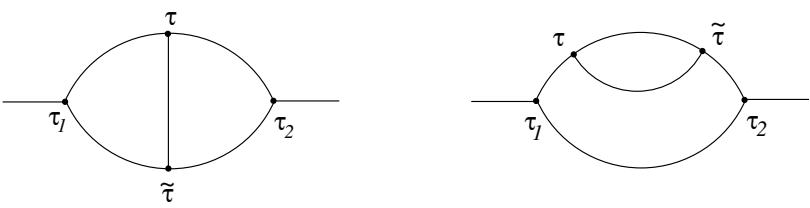

FIG. 5: Two loop diagrams in the $\phi^{3}$ theory with internal time coordinates.

On the other hand, in the zero temperature Euclidean theory, the time variable takes values over the entire real line so that a zero temperature graph would have the internal time coordinate integrated over

$$
\int_{-\infty}^{\infty} \mathrm{d} \tau
$$

So, in order to establish a relation between finite temperature graphs and the zero temperature ones, one has to somehow extend the range of integration of the internal time coordinates at finite temperature to the entire real line as well.

When there is integration over only a single internal time coordinate, it can be shown explicitly that

$$
\begin{aligned}
& \int_{0}^{\frac{1}{T}} \mathrm{~d} \tau \prod_{i=1}^{N} \Delta^{(T)}\left(\tau-\tau_{i}, E_{i}\right) \\
& =O^{(T)} \int_{0}^{\frac{1}{T}} \mathrm{~d} \tau \prod_{i=1}^{N} \Delta^{(T=0)}\left(\tau-\tau_{i}, E_{i}\right) \\
& =O^{(T)} \int_{-\infty}^{\infty} \mathrm{d} \tau \prod_{i=1}^{N} \Delta^{(T=0)}\left(\tau-\tau_{i}, E_{i}\right) .
\end{aligned}
$$

Namely, the extra terms needed to extend the range of the integration are annihilated by the thermal operator (they are not zero by themselves). The same feature also arises explicitly when there are integrations over two internal time coordinates. In fact, in general, we can prove that the range of the integration $\left(0, \frac{1}{T}\right)$ for any internal time coordinate can be extended to $(-\infty, \infty)$ under the action of the thermal operator. I refer the reader to [1] for the proof which is a bit involved. As we will see in the next section, the proof of a relation between finite temperature graphs and the zero temperature ones is most direct in the closed time path formalism.

As a result, it follows that independent of whether there are internal time coordinates (that need to be integrated) in a Feynman graph or not, a finite temperature Feynman graph can be factorized into the corresponding zero temperature graph and a thermal operator which we call the thermal operator representation for a finite temperature graph. Explicitly, if we have diagram corresponding to a $\mathrm{N}$-point function at any loop, we can write

$$
\begin{aligned}
\Gamma_{N}^{(T)} & =\int \prod_{i=1}^{I} \frac{\mathrm{d}^{3} k_{i}}{(2 \pi)^{3}} \prod_{v=1}^{V}(2 \pi)^{3} \delta_{v}^{(3)}(k, p) \gamma_{N}^{(T)} \\
& =\int \prod_{i=1}^{I} \frac{\mathrm{d}^{3} k_{i}}{(2 \pi)^{3}} \prod_{v=1}^{V}(2 \pi)^{3} \delta_{v}^{(3)}(k, p) O^{(T)} \gamma_{N}^{(T=0)},
\end{aligned}
$$

where $I$ denotes the number of internal propagators and $V$ the number of interaction vertices in the graph. Furthermore, we have identified the thermal operator for the graph as

$$
O^{(T)}=\prod_{i=1}^{I} O^{(T)}\left(E_{i}\right)
$$

We also note that since the thermal operator is independent of the time variable, we can now Fourier transform the time variables to go to the energy-momentum space and the factorization continues to hold. In other words, the sum over the Matsubara frequencies for a finite temperature graph can be obtained from the integrals over energies of the corresponding zero temperature graph of the Euclidean field theory under the action of the thermal operator.

This factorization of a finite temperature graph in terms of the corresponding zero temperature one is a powerful result, but this raises some interesting questions. For example, one wonders how such a relation is possible. After all, at finite temperature, a Feynman diagram represents an ensemble average of a time ordered correlation function whereas at zero temperature a diagram is related to the vacuum expectation value. Therefore, for a relation to exist between the two, we must have

$$
\left\langle\mathcal{T}\left(\prod_{i=1}^{N} \phi\left(\tau_{i}, \vec{p}_{i}\right)\right)\right\rangle_{T} \sim\left\langle 0\left|\mathcal{T}\left(\prod_{i=1}^{N} \phi\left(\tau_{i}, \vec{p}_{i}\right)\right)\right| 0\right\rangle .
$$

Clearly, this is possible only if the expectation value of any product of operators in any state is proportional to the vacuum expectation value. We cannot show this in general to be true. However, we can give some plausibility arguments in support of this by looking at the tree level propagator of the theory.

Let us consider a free scalar field in Euclidean space. In the mixed space we can have the field decomposition of the form

$$
\phi(\tau, \vec{p})=\frac{1}{\sqrt{2 E}}\left(e^{-E \tau} a(\vec{p})+e^{E \tau} a^{\dagger}(-\vec{p})\right),
$$

where the annihilation and the creation operators, $a, a^{\dagger}$, satisfy the usual commutation relations. Given this, it can be directly checked that in the $N$-quantum state

$$
\begin{aligned}
& \left\langle N, \vec{p}\left|T\left(\phi\left(\tau_{1}, \vec{p}_{1}\right) \phi\left(\tau_{2}, \vec{p}_{2}\right)\right)\right| N, \vec{p}\right\rangle=\delta^{(3)}\left(\vec{p}-\vec{p}_{1}\right) \\
& \times\left(1+N\left(1-S\left(E_{1}\right)\right)\left\langle 0\left|T\left(\phi\left(\tau_{1}, \vec{p}_{1}\right) \phi\left(\tau_{2}, \vec{p}_{2}\right)\right)\right| 0\right\rangle .\right.
\end{aligned}
$$

Namely, the expectation value of the time ordered product of two fields in any $N$ quantum state is proportional to the vacuum expectation value of the same. Furthermore, summing 
over all such states with the Boltzmann weight factor leads to basic the thermal operator representation for the propagator derived earlier.

One can similarly wonder about the meaning of such a relation and the meaning of the thermal operator. To understand the meaning of the thermal operator better, let us note that the basic thermal operator for the propagator can be checked to correspond to a projection operator,

$$
\left(O^{(T)}(E)\right)^{2}=O^{(T)}(E) .
$$

Therefore, it begs the question as to what does it project onto. This is easily understood by noting that the finite temperature Feynman propagator can be written as

$$
\Delta^{(T)}(\tau, E)=O^{(T)}(E) \Delta^{(T=0)}(\tau, E) .
$$

Since the zero temperature propagator has no particular periodicity properties while the finite temperature propagator does, this shows that the basic thermal operator projects onto the space of periodic functions (for bosons). This, in turn, yields an ensemble average.

There are other interesting properties that the basic thermal operator satisfies. Let me simply note one other. It can also be checked that

$$
O^{\left(T_{2}\right)}(E) O^{\left(T_{1}\right)}(E)=O^{\left(T_{1}\right)}(E) .
$$

Namely, the application of a second thermal operator at a different temperature has no effect. This may seem strange at first. However, this is exactly what one would expect from the property of the thermal operator described earlieer. Namely, once the application of the first thermal operator has produced an ensemble average, the second operator can only average the identity operator over the ensemble leading to nothing new.

\section{CLOSED TIME PATH FORMALISM}

As we have mentioned earlier, in the real time formalism known as the closed time path formalism, the proof of the factorization of finite temperature Feynman graphs is most direct. Let us recall that in the closed time path formalism (as in any real time formalism), the matrix has a $2 \times 2$ matrix structure. For the real, massive scalar field, the components of the propagator matrix were already given in the momentum space. Fourier transforming this to the mixed space (namely, Fourier transforming the energy variable), it is easy to check that the basic finite temperature propagator again factorizes. What is more impressive is that although the propagator is a matrix, each component of the matrix factorizes by the same scalar thermal operator so that we can write

$$
\begin{aligned}
& \Delta_{a b}^{(T)}(t, E) \\
& \quad=(1+n(E)(1-S(E))) \Delta_{a b}^{(T=0)}(t, E) \\
& \quad=O^{(T)}(E) \Delta_{a b}^{(T=0)}(t, E), \quad a, b= \pm .
\end{aligned}
$$

The basic thermal operator is exactly the same as the one we had already derived earlier in the imaginary time formalism.
The further simplicity of the real time formalism is that in this formalism, time takes values over the entire real axis both at zero as well as at finite temperature. Consequently, when we have internal time coordinates in a Feynman graph, we do not worry about extending the range of integration (as we did in the imaginary time formalism). As a result, the thermal operator representation follows directly even when there are internal time coordinates. Indeed, in this formalism, the proof of thermal operator representation for any finite temperature Feynman graph is the most direct. It is worth noting here that even though thermofield dynamics corresponds to the other commonly used real time formalism, the finite temperature propagator in thermofield dynamics only has a matrix factorization (unlike the scalar factorization for the closed time path formalism) and as such the thermal operator representation is hard to prove.

Since the thermal operator representation in the closed time path formalism is so simple, we will now discuss other theories in this formalism. First, we note that if we have fermion theory without a chemical potential, then in the closed time path formalism, it is easy to see in the mixed space that the $2 \times 2$ propagator matrix factorizes through a scalar basic thermal operator as

$$
S_{a b}^{(T)}(t, \vec{p})=O_{\mathrm{F}}^{(T)}(E) S_{a b}^{(T=0)}(t, \vec{p}), \quad a, b= \pm,
$$

where

$$
\begin{aligned}
O_{\mathrm{F}}^{(T)}(E) & =\left(1-n_{\mathrm{F}}(1-S(E))\right), \\
S_{++}^{(T=0)}(t, \vec{p}) & =\frac{1}{2 E}\left[A \theta(t) e^{-i E t}+B \theta(-t) e^{i E t}\right], \\
S_{+-}^{(T=0)}(t, \vec{p}) & =\frac{1}{2 E} B e^{i E t}, \\
S_{-+}^{(T=0)}(t, \vec{p}) & =\frac{1}{2 E} A e^{-i E t}, \\
S_{--}^{(T=0)}(t, \vec{p}) & =\frac{1}{2 E}\left[B \theta(t) e^{i E t}+A \theta(-t) e^{-i E t}\right],
\end{aligned}
$$

with

$$
A=\gamma^{0} E-\vec{\gamma} \cdot \vec{p}+m, \quad B=-\gamma^{0} E-\vec{\gamma} \cdot \vec{p}+m,
$$

and $n_{\mathrm{F}}(E)$ denoting the Fermi-Dirac distribution function. Since this thermal operator is time independent, if we take an interacting theory of scalar and fermion fields, it is clear that any Feynman graph in this interacting theory at finite temperature will factorize in terms of the corresponding zero temperature graph and the thermal operator that would be product of bosonic and fermionic thermal operators depending on the particular graph.

In the same manner, we can also look at a gauge theory in this formalism. For example, if we consider a Yang-Mills theory in the Feynman gauge, we know that the gauge propagator is given by a (massless) scalar propagator up to a trivial Lorentz structure and the internal symmetry structure. Since we know that in the mixed space the $2 \times 2$ finite temperature matrix propagator for a scalar field in the closed time path formalism factorizes through a scalar thermal operator, it follows 
that for the gauge field in the Feynman gauge, we can write

$$
\begin{aligned}
& D_{\mu v, a b}^{\alpha \beta(T)}(t, E)=-\eta_{\mu v} \delta^{\alpha \beta} \Delta_{a b}^{(T)}(t, E) \\
& \quad=O^{(T)}(E) D_{\mu v, a b}^{\alpha \beta(T=0)}(t, E), \quad a, b= \pm .
\end{aligned}
$$

In any other gauge, only the Lorentz structure multiplying the scalar propagator modifies and, consequently, the factorization continues to hold. Similarly, the ghost propagator is a scalar propagator which we know factorizes and so in the Yang-Mills theory all the $2 \times 2$ finite temperature matrix propagators factorize through the same basic thermal operator. Yang-Mills theory, on the other hand, is different in the sense that it is an interacting theory and in the mixed space, it has derivative interactions. However, since the basic thermal operator is independent of time, derivatives commute with the thermal operator and the thermal operator representation holds for Yang-Mills theory as well as for QCD (interacting gauge fields and fermions). This shows, therefore, that the thermal operator representation holds for any interacting theory (scalar, fermion and gauge) at finite temperature and zero chemical potential. We will take up the interesting issue of theories at finite temperature and finite chemical potential in the next section.

\section{FERMIONS (WITH CHEMICAL POTENTIAL)}

The systems at finite temperature with a chemical potential are, in general, harder to handle. The chemical potential can be thought of as a coupling to a background electrostatic potential. At zero temperature its effect is to introduce a trivial phase factor (depending on the chemical potential) into the components of the $(2 \times 2)$ matrix propagator. This is trivial to handle. However, at finite temperature, in addition to this phase factor, the positive and the negative frequency components develop a chemical potential dependent distribution function which makes it harder to handle them. Namely, the positive and the negative frequency terms respond differently to a chemical potential. (Another way of saying is that particles and anti-particles have different distribution functions.) For example, in the case of a complex fermion field at finite temperature and chemical potential, the mixed space $2 \times 2$ matrix propagator in the closed time path formalism takes the form

$$
\begin{aligned}
S_{++}^{(T, \mu)} & =\frac{e^{i \mu t}}{2 E}\left[A\left(\theta(t)-n_{-}\right) e^{-i E t}+B\left(\theta(-t)-n_{+}\right) e^{i E t}\right], \\
S_{+-}^{(T, \mu)} & =\frac{e^{i \mu t}}{2 E}\left[-A n_{-} e^{-i E t}+B\left(1-n_{+}\right) e^{i E t}\right], \\
S_{-+}^{(T, \mu)} & =\frac{e^{i \mu t}}{2 E}\left[A\left(1-n_{-}\right) e^{-i E t}-B n_{+} e^{i E t}\right], \\
S_{--}^{(T, \mu)} & =\frac{e^{i \mu t}}{2 E}\left[A\left(\theta(-t)-n_{-}\right) e^{-i E t}+B\left(\theta(t)-n_{+}\right) e^{i E t}\right]
\end{aligned}
$$

where $n_{ \pm}=n_{\mathrm{F}}(E \pm \mu)$. Therefore, it is not clear whether the thermal propagator will factorize in this case. However, a little bit of work shows that in spite of this complex structure, the propagator has a thermal operator representation given by

$$
S_{a b}^{(T, \mu)}(t, \vec{p})=e^{i \mu t} O_{\mathrm{F}}^{(T, \mu)}\left(E, \partial_{t}\right) S_{a b}^{(T=0=\mu)}(t, \vec{p}),
$$

where $a, b= \pm$ and

$$
\begin{gathered}
O_{\mathrm{F}}^{(T, \mu)}\left(E, \partial_{t}\right)=1-\frac{n_{+}+n_{-}}{2}(1-S(E)) \\
+\frac{n_{+}-n_{-}}{2}(1+S(E)) \frac{1}{E} i \partial_{t} .
\end{gathered}
$$

This is the factorization one obtains if we insist on restricting ourselves to the use of the reflection operator, $S(E)$, as has we have done in the earlier discussions. We note that when $\mu=0$, this thermal operator reduces to the one described earlier for the fermions. It can also be checked that in spite of its complex form, this thermal operator continues to be a projection operator. However, its basic structure is different from the earlier ones in the following way. Although it still does not depend on the time coordinates, it now depends on time derivative (basically, this reflects the asymmetry in particles and anti-particles and vanishes when $\mu=0$ ). It is because of the presence of this derivative operator that it is no longer possible to give a thermal operator representation for an arbitrary graph in terms of this basic thermal operator. (Mainly, we cannot take out the derivative terms out of integrals involving internal time coordinates in a simple manner.)

On the other hand, if we allow for other operators besides $S(E)$ in defining a factorization for the thermal propagator, one can avoid this difficulty. In particular, if we introduce a distribution operator, $\hat{N}^{(T, \mu)}(E)$ such that acting on the positive and the negative frequency terms, it gives the appropriate distribution functions [3]

$$
\hat{N}_{\mathrm{F}}^{(T, \mu)}(E) e^{\mp i(E \mp \mu) t}=n_{\mp}(E) e^{\mp i(E \mp \mu) t},
$$

then the thermal propagator can be factorized with a thermal operator that is independent of time as well as time derivatives. With this, one can give a thermal operator representation for any arbitrary graph at finite temperature and chemical potential.

As an application of this, one can calculate the fermion selfenergy in QED at one loop at finite temperature and chemical potential. Even in the absence of a chemical potential, such a calculation is highly nontrivial and the analysis of the poles of the fermion propagator is involved. In the presence of a chemical potential, the analysis of the pole of the fermion propagator leads to the fact that the tree level chemical potential renormalizes at finite temperature [2]. It is, of course, a finite renormalization. At very high temperature $(T \gg m$ where $m$ denotes the tree level fermion mass), the pole occurs at

$$
m_{\mathrm{F}}=\frac{e T}{2 \sqrt{2}}, \quad \mu_{\mathrm{R}}=\mu\left(1-\frac{e^{2}}{16 \pi^{2}}\right) .
$$

The thermal mass of the fermion is well known, however, the renormalization of the chemical potential, to the best of our knowledge, has not been discussed in the literature. From the point of view of the Lagrangian density, the chemical potential represents a parameter that, in principle, can be renormalized 
and we see that the renormalization has a screening behavior much like one would expect from the renormalization of an Abelian charge (remember that an alternate way of viewing the chemical potential is to think of this as a coupling to a background electrostatic field).

\section{SUMMARY}

In this talk I have tried to describe to you a very beautiful relation between the finite temperature Feynman graphs and the corresponding zero temperature graphs, both in the imaginary time formalism and the real time (closed time path) formalism. Such a relation holds for any interacting theory with scalars, fermions and Yang-Mills fields with or without a chemical potential. In addition to being of computational use, the thermal operator representation is a powerful result which allows one to study many aspects of thermal field theories in a direct manner. One can, for example, study the cutting rules at finite temperature and chemical potential $[4,8,12]$ in a sim- pler manner from the behavior at zero temperature. Similarly, questions such as gauge invariance, divergence structure in thermal field theories can be investigated from the corresponding behaviors in the zero temperature theory. One may be able to give a simpler derivation of the forward scattering amplitude method at finite temperature through the application of the thermal operator and hard thermal loops may be easier to understand from this point of view. One may be able to derive a renormalization group flow of thermal amplitudes with respect to temperature directly since the temperature dependence of a graph is contained entirely in the thermal operator. Of course, there are many other possible questions that one can think of which can be analyzed in a simpler manner in this framework.

Acknowledgment: This work was supported in part by US DOE Grant number DE-FG-02-91ER40685.
[1] F. T. Brandt, A. Das, O. Espinosa, J. Frenkel, and S. Perez, Physical Review D 72, 085006 (2005).

[2] F. T. Brandt, A. Das, O. Espinosa, J. Frenkel, and S. Perez, Physical Review D 73, 065010 (2006).

[3] F. T. Brandt, A. Das, O. Espinosa, J. Frenkel, and S. Perez, Physical Review D 73, 067702 (2006).

[4] F. T. Brandt, A. Das, O. Espinosa, J. Frenkel, and S. Perez, Physical Review D 74, 085006 (2006).

[5] T. Matsubara, Progr. Theo. Phys. 14, 351 (1955).

[6] J. Kapusta, Finite Temperature Field Theory, (Cambridge University Press, 1989).

[7] M. Le Bellac, Thermal Field Theory, (Cambridge Univesrity Press, 1996).
[8] A. Das, Finite Temperature Field Theory, (World Scientific, 1997).

[9] J. Schwinger, J. Math. Phys. 2, 407 (1961).

[10] K. T. Mahanthappa and P. M. Baxi, J. Math. Phys. 4, 1 (1963); L. V. Keldysh, Zh. Eksp. Teor. Fiz. 47, 1515 (1964) [Sov. Phys. JETP 20, 1018 (1965)].

[11] Y. Takahashi and H. Umezawa, Collective Phenomena 2, 55 (1975); H. Umezawa, H. Matsumoto, and M. Tachiki, Thermofield Dynamics and Condensed States, (North-Holland, 1982).

[12] P. F. Bedaque, A. Das, and S. Naik, Mod. Phys. Lett. A12, 2481 (1997). 